\title{
Intraoperative Intravenous Lidocaine
}

\author{
Caio Marcio Barros de Oliveira, TSA ${ }^{1}$, Adriana Machado Issy ${ }^{2}$, Rioko Kimiko Sakata, TSA ${ }^{3}$
}

Summary: Oliveira CMB, Issy AM, Sakata RK - Intraoperative Intravenous Lidocaine.

Background and objectives: Most patients undergoing surgery experience moderate to severe pain, indicating the need to improve the anesthetic technique. Intravenous lidocaine has been widely used in the treatment of chronic pain. The objective of this report was to review the use of intravenous lidocaine for postoperative analgesia.

Contents: The pharmacologic aspects and mechanism of action of lidocaine as well as clinical studies in which the authors used intraoperative lidocaine were reviewed.

Conclusions: Intravenous lidocaine can promote analgesia in surgical procedures, representing another alternative for the treatment of acute pain. Controlled studies with different surgical interventions could bring more information on this modality of analgesia.

Keywords: ANESTHETICS, Local: lidocaine; PAIN, Postoperative.

[Rev Bras Anestesiol 2010;60(3): 325-333] @Elsevier Editora Ltda.

\section{INTRODUCTION}

Postoperative pain is a unique and common type of acute pain. Several studies have indicated that appropriate pain treatment protocols reduce postoperative morbidity, improve the results of the surgery, and decrease hospital costs 1,2 . Besides, adequate relief of postoperative pain is associated with positive long-term effects for patients, such as: reduced postoperative cognitive changes, better quality of life, and reduced risk of chronic or persistent postoperative pain ${ }^{3,4}$. However, it was recently demonstrated that approximately $50 \%$ to $70 \%$ of patients undergoing surgeries experience moderate to severe pain, indicating that, despite the development of new drugs and implementation of new analgesic techniques, postoperative pain still is poorly evaluated and treated ${ }^{5,6}$. The lack of knowledge of the pathophysiology of postoperative pain is an important cause of this problem ${ }^{7}$.

Surgical injury leads to hemodynamic, metabolic, and immunologic changes, which are mediated by endogenous substances known as cytokines ${ }^{8}$, polypeptides or glycoproteins produced by several types of cells, at the site of injury, and the immune system, being responsible for the inflammatory response in sites of infection or injury and

Received from the Discipline of Anesthesiology, Pain and Intensive Care of the Escola Paulista de Medicina da Universidade Federal de São Paulo (EPM/UNIFESP), SP

1. Anesthesiologist; Specialist in Pain at the EPM/UNIFESP; Master's degree in Medicine 2. Adjunct Professor of the Discipline of Anesthesiology, Pain and Intensive Care of the EPM/UNIFESP

3. Coordinator of the Sector of Pain and Intensive Care of the EPM/UNIFESP

Submitted on February 9, 2010

Approved on February 22, 2010

Correspondence to:

Dra. Rioko Kimiko Sakata

Rua Três de Maio, 61

Vila Clementino

04044-020 - São Paulo, SP, Brasil

Email: riokoks.dcir@epm.br promotion of tissue healing ${ }^{10}$. Besides, activated glial cells in the surgical wound stimulate the production of cytokines in the central nervous system, which can induce peripheral and central sensitization through nitrous oxide, free radicals, and excitatory amino acids, and possibly chronic and neuropathic pain ${ }^{3,9}$.

An exaggerated and persistent production of cytokines that can cause damage of target organs, multiple organ failure, and death is seen after severe trauma or infection ${ }^{10}$.

Interleukin-6 (IL-6), a molecule of approximately 21,000 Daltons, is a cytokine detected early in the process whose increased levels are associated more with the degree of tissue injury during surgery than with the duration of the procedure 8,11-13. It has been considered a predictor of the severity of the trauma up to six hours after admission to the hospital ${ }^{12}$. It induces the hepatic synthesis of acute phase proteins like Creactive protein (CRP). The level of CRP reflects the impact of the trauma and is associated with the extension of damaged tissue, but it can only be detected after six hours ${ }^{11,12}$. After surgical damage, IL- 6 is detected after 60 minutes with peak blood concentration between 4 and 6 hours, and it can persist for up to 10 days 8,14 .

Intravenous and epidural lidocaine promote significant relief of postoperative pain, with a reduction in severity, decreased consumption of inhalational anesthetics and opioids, early recovery of bowel function, and decreased production of interleukins ${ }^{3,13,14}$. Lidocaine has significant anti-inflammatory properties ${ }^{15}$, reducing the in vitro and in vivo release of cytokines by reducing neutrophil activation 16-19.

The analgesic effects of lidocaine in surgical trauma can be due to blockade of the neuronal transmission at the site of injury, attenuating the neurogenic response, and by the intrinsic systemic anti-inflammatory properties. Besides, depending on the dose, lidocaine can reduce cytokine-induced cellular damage through mechanisms that involve mitochondrial adenosine triphosphate (ATP)-gated potassium channels $3,15,19$. 
The analgesic properties of lidocaine can persist even after the reduction of its plasma levels, favoring the theory of the blockade of nervous conduction ${ }^{14}$.

There are very few studies on the intraoperative use of intravenous lidocaine in the literature, and that triggered our interest on the subject.

\section{Lidocaine: Pharmacological Aspects}

Lidocaine [2-(diethylamino)-N-(2,6-dimethylphenyl)acetamide] is the local anesthetic used more often, and it is considered the prototype of amino-amide local anesthetics ${ }^{20,21}$. It is a weak base (ionization constant pKa 7.9), and poorly hydrosoluble.

Voltage-gated sodium channels (Nav) represent the classical targets of lidocaine. They are formed by a complex of glycosilated proteins with one $\varangle \alpha\left(260,000\right.$ Daltons), and $\beta_{1}$ to $\beta_{4}$ (33,000 to 38,000 Daltons) subunits. The $\alpha$ subunit has four homologous domains (1 to 4), each one composed of six transmembrane helicoidal segments ( $S 1$ to $S 6$ ) and a nonhelicoidal region between S5 and S6 ( $P$ segment), where the sodium channel is located ${ }^{22,23}$. In mammals, voltage-gated sodium channels have nine different types (isotypes) of $\alpha$ subunits (Nav 1.1 to 1.9), and some of them are related with neuropathic pain ( $\operatorname{Nav} 1.3,1.7,1.8$, and 1.9) and others with inflammatory pain (Nav 1.7, 1.8, and 1.9) ${ }^{25}$.

After crossing the neuronal membrane, the intracellular $\mathrm{pH}$ converts lidocaine into its ionized form, which has an irreversible effect on the S6 portion of domain 4 of the $\alpha$ subunit inside voltage-gated sodium channels ${ }^{23,24}$. As the action of the anesthetic drug develops the threshold for electrical excitability increases gradually, the peak of the action potential decreases, conduction of neuronal impulse slows down, and the safety factor for conduction decreases. This reduces the probability of propagation of the action potential and nerve conduction fails ${ }^{22}$. Sensorial loss is progressive and in the following order: pain, temperature, touch, deep pressure, and motor function, according to the diameter of the nerve fibers ${ }^{24}$.

The affinity of lidocaine for sodium channels varies according to the conformation of the channel, being greater when the channel is opened (active or inactive) and lower when it is closed (deactivated or at rest). Thus, the amount of molecules of ionized lidocaine that have access to the site of action increases with higher frequencies of neuronal stimulation, increasing the degree of the blockade (use-dependent or frequency-dependent blockade) 21,24 .

Lidocaine is also analgesic and anti-inflammatory when acting in potassium channels, calcium channels, and protein G-coupled receptors $21,24,25$.

In clinical practice, lidocaine can be used in different forms (i.e., solution, eye drops, cream) and different administration routes (i.e., epidural, subarachnoid, intrapleural, intravenous, intramuscular, intra-articular, topical) ${ }^{21}$, but here we will focus on the intravenous administration.

Lidocaine is metabolized in the liver, by the microsomal enzymatic system (cytochrome P450), with clearance rate of
$0.85 \mathrm{~L} . \mathrm{kg}^{-1} \cdot \mathrm{h}^{-1}$. It is oxidized to monoethylglycine xylidide and a portion of this substrate is hydrolyzed to glycine xylidide. Those metabolites are active and they have been implicated in cases of intoxication after repeated doses or continuous infusion. It is eliminated by the kidneys, with a fast elimination phase, of 8 to 17 minutes, and a slow phase, of 87 to 108 minutes. Less than $10 \%$ of lidocaine is eliminated unchanged in the urine $20,24,26$.

It has been established that systemic absorption of any local anesthetic depends on the degree of vascularization at the injection site, being higher in intravenous, tracheal, intercostal, paracervical, epidural, brachial plexus, sciatic, and cutaneous administration, respectively $21,22,24$

The severity of adverse effects depends on the dose, rate, and site of administration, as well as the physical status of the patient in relation to his/her age, clinical conditions, and pregnancy ${ }^{24}$.

As the concentration of lidocaine in the systemic circulation increases, several signs and symptoms related to the central nervous system and cardiovascular system develop. Analgesia and inhibition of cortical motor neurons have been reported with serum levels lower than $5 \mu \mathrm{g} \cdot \mathrm{mL}^{-1}$, explaining its anticonvulsant action ${ }^{26}$. Perioral paresthesia, metallic taste, dizziness, slurred speech, diplopia, tinnitus, confusion, agitation, muscular spasms, and seizures have been reported with higher levels ${ }^{24}$. Occasionally, seizures represent the first indication of severe intoxication ${ }^{27}$, which is secondary to inhibition of inhibitory neurons by GABA (gamma-aminobutyric acid) receptors in the cerebral amygdala ${ }^{24,26}$. Usually, seizures are seen with plasma levels higher than $8 \mu \mathrm{g} \cdot \mathrm{mL}^{-1}$, but they can also be seen with lower plasma levels in the presence of hypercapnia 20 . Bradycardia with an increase in PR interval and widening QRS complex is seen in cardiovascular toxicity ${ }^{24}$. Allergy to amino amides is considered extremely rare, with an estimated rate of $1 \%$ of recorded reactions ${ }^{24}$.

Treatment of the intoxication includes supportive measures with oxygenation, hydration, and the use of vasopressors, inotropic drugs, anticonvulsant, and antiarrhythmics as needed ${ }^{24}$.

\section{Intravenous Lidocaine}

Intravenous lidocaine is widely used ${ }^{28}$. After intravenous administration, it is initially distributed to highly vascularized organs, such as brain, kidneys, and heart, and then to less vascularized organs such as skin, skeletal muscles, and adipose tissue. It has a high volume of distribution (91 L. $\mathrm{kg}^{-1}$ ), and an oil/water partition coefficient of 366 , with intermediate potency. Around $60 \%$ of its molecules are bound to plasma proteins, especially $\alpha 1$-acid glycoprotein ${ }^{24}$.

Approximately $40 \%$ of intravenous lidocaine is temporarily extracted during first pass through the lungs, where the $\mathrm{pH}$ is lower than that of the plasma. Consequently, this reduces the chances of intoxication in cases of accidental intravenous administration ${ }^{24,27}$. About $90 \%$ of intravenous lidocaine undergo hepatic metabolism, and it has a half-life of 1.5-2 hours. 
Intravenous lidocaine has peripheral and central actions, and the mechanisms include: blockade of sodium channels, glycinergic action, blockade of NMDA receptors, and reduction in substance $P{ }^{29}$. Low concentrations of lidocaine inhibit abnormal activity in primary afferent fibers especially C- fibers, cause sympathetic blockade and vasodilation, and break the vicious cycle responsible for pain maintenance.

Sodium channel blockade causes inhibition of spontaneous and evoked neuronal activity ${ }^{30}$. It reduces neuronal hyperactivity, leading to pain relief 30,31 . In therapeutic concentrations, lidocaine reduces hyperexcitability without affecting nerve conduction.

Intravenous lidocaine promotes a reduction in spinal cord sensitivity ${ }^{32}$. It reduces the activity of spinal cord neurons, and it decreases post-synaptic depolarization mediated by $\mathrm{N}$-methyl-D-aspartate (NMDA) and neurokinin receptors ${ }^{29}$. It can reduce glutamate activity in the dorsal horn of the spinal cord, and its effect is greater in a subgroup of spinal cord neurons ${ }^{30}$. The higher susceptibility of hyperexcitable neurons to lidocaine can be explained by changes in the expression of sodium channels in the damaged nerve, subjecting them to exaggerated lidocaine-induced blockade ${ }^{29}$. This action is related with the suppression of ectopic discharges caused by changes in sodium channels ${ }^{29}$.

Lidocaine promotes significant pain relief with reduction in allodynia and hyperalgia 32,33 . A reduction in spontaneous pain, dysesthesia, mechanical hyperalgia, and mechanical allodynia is seen ${ }^{29,34}$.

Preferential blockade of inactive sodium channels guarantees that only hyperexcitable sodium channels, such as those with ectopic activity after nerve damage that are permanently depolarized, are blocked ${ }^{35}$.

Lidocaine does not seem to be as effective in nociceptive pain ${ }^{33}$. This effect seems to be dose-dependent, and $5 \mathrm{mg} \cdot \mathrm{kg}^{-1}$. 30 minutes was considered the dose with the most consistent response ${ }^{35}$. In another study, a correlation between maximal plasma concentration and maximal pain relief was not observed $^{29}$.

Intravenous lidocaine should not be used in patients with arrhythmias, heart failure, coronary artery disease, AdamsStokes, or heart blocks ${ }^{29}$. Caution should be taken when using lidocaine in patients with hepatic failure, sinus bradycardia, and incomplete branch block ${ }^{29}$.

The most common side effects are usually related to the CNS 29. Patients might develop: somnolence, dizziness, metallic taste, headache, blurred vision, paresthesia, dysarthria, euphoria, and nausea ${ }^{29,33}$. Fast administration of higher doses can cause tinnitus, weakness, tremor, and agitation. Cardiovascular changes are usually minimal with usual doses.

\section{Intravenous Lidocaine and postoperative pain}

Several authors have demonstrated that low doses of intravenous lidocaine (plasma concentration below $5 \mu \mathrm{g} \cdot \mathrm{mL}^{-1}$ ) attenuate pain after procedures, without interfering with normal nerve conduction, with a lower incidence of side effects ${ }^{14,36-43}$. The best dose of lidocaine to obtain greater efficacy of postoperative pain treatment has not been defined 44 , probably because central and peripheral sensitization patterns differ according to the type and site of the surgery 40

Intravenous lidocaine has analgesic ${ }^{39}$, anti-hyperalgic ${ }^{31,38,39}$, and anti-inflammatory ${ }^{15}$ properties, and it is capable of reducing intra- and postoperative analgesic requirements and the length of hospitalization ${ }^{14,39,41,42}$. Its effects are more pronounced when the infusion is administered during the intraoperative period ${ }^{39}$ and they can continue for days or weeks, i.e., beyond the infusion time and plasma half-life ${ }^{43,45}$, indicating that it affects other targets, and not only voltage-gated sodium channels, and it suggests a prevention of the hypersensitivity of the central or peripheral nervous system or both ${ }^{41}$.

An experimental study demonstrated that intravenous lidocaine produces three distinct phases of relief: the first develops during the infusion and it decreases 30 to 60 minutes after administration; the second is a phase of transition and it is seen approximately 6 hours after the infusion; and the third phase is seen 24 to 48 hours after the infusion and continues for 21 days 46

Besides affecting voltage-gated sodium channels (Nav), especially isotypes Nav 1.7, 1.8, and 1.9, present in nociceptors in inflamed tissues ${ }^{45}$, lidocaine affects $\mathrm{G}$ protein-coupled receptors (GPCR), NMDA (N-methyl-D-aspartate) receptors, and potassium and calcium channels, interfering with the conduction of excitatory impulses on A-delta and $\mathrm{C}$ fibers, visceral pain, central sensitization, and immune response 15,19,36,38,42,44,47,48. It seems that lidocaine causes direct blockade of NMDA receptors by inhibiting protein kinase C (PKC) ${ }^{47}$, exerting significant influence on postoperative hyperalgia and tolerance to opioids $31,38,49$

Lidocaine interferes, via GPCR with some inflammatory processes, such as neutrophil sensitization and lysosomal degranulation, production of free radicals, and secretion of cytokines by macrophages and glial cells 15,46,50,51.

Although lidocaine has less affinity for voltage-gated potassium and calcium channels, it also affects them. Usually, it blocks opened ion channels, similar to the susceptible state of sodium channels. Inhibition of calcium channels in pre-synaptic nerve endings affects, significantly, the propagation of pain impulses ${ }^{48}$. It has been hypothesized that lidocaine reduces cytokine-induced cellular damage using mitochondrial adenosine triphosphate (ATP)-gated potassium channels ${ }^{19}$.

\section{Clinical Studies}

The synergic action of intravenous lidocaine $\left(3 \mathrm{mg} \cdot \mathrm{kg}^{-1} \cdot \mathrm{h}^{-1}\right)$ and intramuscular dextromethorphan $(40 \mathrm{mg}$ ) on postoperative pain relief and recovery of intestinal peristalsis was seen in videolaparoscopic cholecystectomy when those drugs were administered 30 minutes before surgery ${ }^{40}$. 
A reduction in postoperative pain severity and morphine consumption, with apparent prevention of central hyperalgia, was observed in patients undergoing abdominal surgeries when intravenous lidocaine (bolus of $1.5 \mathrm{mg} \cdot \mathrm{kg}^{-1}$, followed by infusion of $\left.1.5 \mathrm{mg} \cdot \mathrm{kg}^{-1} \cdot \mathrm{h}^{-1}\right)$ was administered 50 minutes before the surgical incision, and maintained for up to 60 minutes after skin closure, and this benefit was more evident 36 hours after surgery ${ }^{39}$.

The intravenous administration of lidocaine, pre-incision bolus of $2 \mathrm{mg} \cdot \mathrm{kg}^{-1}$ and infusion of $3 \mathrm{mg} \cdot \mathrm{kg}^{-1} \cdot \mathrm{h}^{-1}$ until the end of the surgery, promoted significant pain relief and faster return of intestinal function, reduced consumption of the volatile anesthetic and opioid, and attenuated the production of interleukins 1RA (receptor antagonist), 6, and 8 (IL-1RA, IL-6, and IL-8) for 72 hours after conventional colectomy ${ }^{14}$. Similarly, the analgesic profile of lidocaine was clear in patients undergoing videolaparoscopic colectomy ${ }^{41}$.
On the other hand, intravenous bolus of lidocaine, 1.5 $\mathrm{mg} \cdot \mathrm{kg}^{-1}$, and infusion of $1.5 \mathrm{mg} \cdot \mathrm{kg}^{-1} \cdot \mathrm{h}^{-1}$ for up to 60 minutes after skin closure did not produced any increase in analgesia or functional recovery and it did not increase the threshold of tactile pain and pressure after total hip arthroplasty 52 .

\section{CONCLUSION}

Studies have demonstrated that intravenous lidocaine can promote analgesia in surgical procedures, representing another alternative for the treatment of acute pain, especially in patients who cannot undergo spinal anesthesia or those who refuse it. Further controlled studies with different surgical procedures could bring more information on this analgesic modality. 